\title{
Anabases
}

ANABASES Traditions et réceptions de l'Antiquité

\section{Les archives Alfred Foucher (1865-1952) de la Société asiatique (Paris)}

Annick Fenet

\section{(2) OpenEdition}

1 Journals

Édition électronique

URL : http://journals.openedition.org/anabases/2520

DOI : 10.4000/anabases.2520

ISSN : 2256-9421

Éditeur

E.R.A.S.M.E.

\section{Édition imprimée}

Date de publication : 1 mars 2008

Pagination : 163-192

ISSN : 1774-4296

\section{Référence électronique}


Anabases 7 (2008), p. 163-192.

\section{Les archives Alfred Foucher \\ (1865-1952) \\ de la Société asiatique (Paris) 1}

ANNICK FENET

« L'IndE ANGLAISE à la fin du XIXe siècle, c’était celle de la grande époque victorienne [...]. C'était celle dont Rudyard Kipling, le fils du conservateur du Musée de Lahore, écrivait la chronique un tantinet scandaleuse avec une verve si incisive [...]. C'était celle des palais enchantés, des fêtes somptueuses, des danses des bayadères et des jongleries des charmeurs de serpents, des combats d'éléphants et des chasses au tigre [...]. C'était celle où survivait la dernière grande religion naturaliste à la façon de notre paganisme classique, [...] celle aussi des profondes métaphysiques panthéistiques [...]. C'était celle des doctrines ésotériques et des rites occultes, celle des yogis et des théosophes, à laquelle l'âme toujours en peine de Pierre Loti allait demander le secret de l'immortalité [...]. C'était celle enfin des entreprises de modernisations, des réseaux de chemins

1 Ce travail s'inscrit dans le cadre du programme AREA (Archives of European Archaeology), dont M. Alain Schnapp, professeur à l'Université de Paris I, dirige la section française (AREA-France) ; sur sa phase actuelle dite AREA IV, voir A. SCHNAPP, "AREA, archives de l'archéologie européenne. Bilan et perspectives”, Nouvelles de l'INHA, juin 2006, p. 2-4. C'est ce programme qui a financé (soit 10 mois de vacations à mi-temps, répartis entre janvier 2006 et février 2007) l'examen inédit de ce fonds d'archives, avec pour objectif son intégration d'ici l'été 2008 dans la base AREA consultable en ligne (www. area-archives.org). Nous tenons à exprimer ici toute notre reconnaissance au directeur d'AREA-France pour cette action particulière et son soutien général. Nous sommes également très redevables à Mme Ève Gran-Aymerich, chercheur auprès de l'Académie des Inscriptions et Belles Lettres et maître en histoire de l'archéologie, à M. Paul Bernard, membre de l'Institut, ancien directeur de la Délégation archéologique française en Afghanistan (DAFA) et à M. Pierre-Sylvain Filliozat, membre de l'Institut (AIBL), vice-président de la Société asiatique, pour leur confiance, leurs conseils et le grand bonheur qu'il m'ont donné de pouvoir travailler sur ce fonds. 
de fer, des gigantesques travaux d'irrigation, des recensements, des statistiques, des paperasses administratives, en un mot de ce qu'en Europe nous appelons le progrès, et tout cela faisait de la grande colonie britannique le modèle du genre. La mode était alors chez nous, vous vous en souvenez peut-être, de chanter sur tous les tons, la supériorité des Anglo-Saxons et leur génie colonisateur était l'un des leitmotiv dans ce concert de louanges...

Cette Inde d'hier et qui nous paraît aujourd'hui préhistorique, je l'ai justement visitée pour la première fois dans les dernières années du siècle dernier. [...] À la manière de grandes cataractes, les guerres mondiales créent dans le cours régulier de l'histoire des dénivellations aussi profondes qu'imprévisibles. Dans l'Inde de 1950 qui reconnaîtrait celle de 1900? Déjà en 1920 lors de ma seconde visite, je ne la reconnaissais plus. $[\ldots] »$

C'est en ces termes qu'au début des années 1950 l'orientaliste et indianiste Alfred Foucher (1865-1952) ${ }^{2}$, dans une conférence donnée à la fin de sa vie ${ }^{3}$, rend compte de sa riche expérience, à la fois érudite et vécue, de l'Asie. En effet, ce membre de l'Institut (Académie des inscriptions et belles-lettres), professeur à la Sorbonne à la chaire des langues et littératures de l'Inde, président de l'Institut de civilisation indienne, directeur d'études à l'EPHE et au passage visiting professor à l'Université de Columbia en 1915, qui fut aussi directeur suppléant puis directeur de l'École française d'Extrême-Orient entre 1901 et 1907, créateur et directeur de la Délégation archéologique française en Afghanistan (DAFA) de 1922 à 1946, premier directeur de la Maison franco-japonaise à Tokyo entre 1925 et 1926, opéra de nombreuses missions archéologiques en Asie (Ceylan, Inde, Afghanistan, Perse, Java, Indochine, Japon) entre 1895 et

2 Notices biographiques sur Alfred Foucher : P. Deschamps, Discours de M. Paul Deschamps, président de l'Académie, à l'occasion de la mort de M. Alfred Foucher, membre de l'Académie, Paris, 1952 ; J. Filliozat, "Alfred Foucher", Journal Asiatique 240 (1952), p. 389-394 ; Ch. PiCARD, "Alfred Foucher (1865-1952)", Artibus Asiae 15 (1952), p. 348351 ; R. FAZY, "Alfred Foucher, 1865 à 1952, et son œuvre", Asiatische Studien 7, 1953, p. 81-98 ; A. MERLIN, Notice sur la vie et les travaux de M. Alfred Foucher membre de l'Académie, Paris, 1954 ; C. CHARLE, Les professeurs de la faculté des lettres de Paris. Dictionnaire biographique II, 1909-1939, Paris, 1986, p. 84-85 ; J. LECLANT (dir.), Institut de France, le second siècle (1895-1995), 3 t., Paris, 1999-2005, t. I, p. 515-516. Sur le contexte à la fois historique et archéologique : È. GRAN-AYMERICH, Naissance de l'archéologie moderne, Paris, 1998 ; N. CHEVALIER, La recherche archéologique française AU MoyenOrient, 1842-1947, Paris, 2002.

3 Cette conférence de Foucher intitulée "L'Inde anglaise" appartenait à une série de huit conférences (peut-être radiophoniques ?) sur "L'Angleterre à la fin du XIXe siècle ", réparties entre divers spécialistes sur autant de sujets distincts (texte manuscrit de la conférence et liste de la série, fonds Alfred Foucher, Société asiatique). Sauf mention contraire, toutes les citations à venir proviennent de documents du fonds Alfred Foucher, pour lesquels il n'existe pas encore de cotes (reconditionnement et catalogage à venir, en partie grâce au financement AREA-France). 
1927 - dont les fameuses fouilles de Bactres (Afghanistan) - et publia de nombreuses études parmi lesquelles le monumental Art gréco-bouddhique du Gandhâra ${ }^{4}$ et l'emblématique La vieille route de l'Inde de Bactres à Taxila 5 .

Ces mots, prononcés plus de 55 ans après sa première mission en Inde en $1895^{6}$, expriment bien le sentiment qu'il ressent alors d'avoir été le témoin privilégié sur plus d'un demi-siècle de la formidable évolution du monde tant occidental qu'oriental, en ce qui concerne à la fois leur histoire contemporaine durant la première moitié du

4 L'art gréco-bouddhique du Gandhâra. Étude sur les origines de l'influence classique dans l'art bouddhique de l'Inde et de l'Extrême-Orient: Tome I. Introduction. Les édifices. Les basreliefs, Paris, 1905 (PEFEO V) ; Tome II, 2 fascicules (1. Les images ; 2. L'histoire. Conclusions), Paris, 1918-1922 (= PEFEO VI, 1) ; t. II, 3e fasc. (Additions et corrections, index), Paris, 1951 (PEFEO VI, 2).

5 A. Foucher, Mme E. BAZIN-FOUCher, La vieille route de l'Inde de Bactres à Taxila, Paris, 1942-1947 (vol. I. Introduction, 1e et 2e parties, 1942 ; vol. II. 3e partie, appendices, 1947) (Mémoires de la DAFA I).

6 Selon les propres termes de Foucher, une mission que "sur la généreuse initiative de l'Académie des inscriptions et belles-lettres, le ministère de l'Instruction publique [lui] a [...] confiée [...] pendant 2 ans, de 1895 à 1897 » et qui l'a mené " à travers l'Inde, de Ceylan au Kachmir et du Bengale à la frontière de l'Afghanistan. [...] Le principal objet de ses études, tant sur le terrain que dans les musées, était la curieuse influence que notre art classique a exercé sur le bouddhisme aux environs de notre ère, dans toute la région du Nord-Ouest de l'Inde ». Le récit de la partie du voyage de novembre 1896 à février 1897 est paru sous le titre "Sur la frontière indo-afghane (extraits du journal de route d'un archéologue)" dans la revue Le Tour du Monde. Journal des voyages et des voyageurs, nouvelle série V, 1899 en 5 épisodes : "La frontière du Bounêr", n 40, 7 octobre 1899 (p. 469-504 du tome V) ; "La frontière du Svat" : n 41, 14 octobre 1899 (p. 481-493) ; "La vallée du Svat" : n 42, 21 octobre 1899 (p. 493-504) ; "Le district de Peshavar" : $n^{\circ} 46,18$ novembre 1899 (p. 541-552) ; "Peshavar" : $\mathrm{n}^{\circ}$ 47, 25 novembre 1899 (p. 553-564). Ces articles ont fait ensuite l'objet d'une publication en livre " avec additions et remaniements »: A. Foucher, Sur la frontière indo-afghane, Paris, 1901. Par ailleurs des comptes rendus ont été donnés de l'ensemble de la mission (A. Foucher, "Compte rendu d'une mission dans l'Inde", CRAI 22 avril 1898, p. 257-264 ; id., "Rapport sur une mission d'études archéologiques et religieuses dans l'Inde (nov. 1895-oct. 1897)", Nouvelles archives des missions scientifiques et littéraires IX, 1899, p. 522-558), ainsi qu'une étude provisoire : "Note sur l'itinéraire de Hiuen-tsang au Gandhâra", Actes du XIe congrès international des Orientalistes (Paris, 1897). 1re section : langues et archéologie des pays ariens, Paris, 1899, p. 93-97 - en réalité, cet article n'a pas été écrit par Foucher, mais c'est un " résumé " que les éditeurs des actes donnent du " mémoire accompagné de cartes, de plans et de photographies " qu'il a envoyé au Congrès depuis l'Inde et qu'a lu alors à sa place Louis Finot ; le texte définitif de cette analyse n'est paru que quelques années plus tard : A. FouCHER, "Notes sur la géographie ancienne du Gandhâra (commentaire à un chapitre de Hiuen-Tsang)”, BEFEO 1 (1901), p. 322-369. Il faut enfin rattacher à ce voyage la petite étude : A. FOUCHER, "Note sur les Miniatures inscrites du ms. népâlais A15 de la Bibliothèque de la Société asiatique du Bengale (XI $\mathrm{I}^{\mathrm{e}}$ siècle)", Journal asiatique 7 (9e série/1896), p. 346-349, dont Foucher envoya le texte à Emile Senart. 
XXe s., le regard du premier sur le second et leurs relations réciproques, les jalousies et rivalités européennes pour la maîtrise de l'Orient. Cette transformation est également remarquable dans le domaine de la connaissance des cultures et civilisations asiatiques présentes et passées, dont Foucher a vécu le passage d'une approche littéraire et pour ainsi dire mystique à des voyages d'exploration scientifique et à des études spécialisées 7 .

C'est dire l'importance, pour l'histoire de l'archéologie, du fonds d'archives d'Alfred Foucher conservé à la Société asiatique, couvrant l'ensemble de ses activités et de sa biographie. À travers lui, se dessine une vision plus large de l'orientalisme français de la III République et de son évolution après la Seconde Guerre mondiale, et ce à travers ses acteurs, ses institutions tant en métropole que dans les colonies ou à l'étranger, ses champs d'actions et ses méthodes.

Au terme d'une première phase de travail de dépouillement et d'inventaire systématiques des documents papier, le présent article tend à offrir une présentation générale de l'ensemble du fonds Alfred Foucher et de ses principaux centres d'intérêt, avant nous l'espérons d'autres publications thématiques à venir. Le lecteur voudra donc bien nous excuser si nous ne ferons ici qu'effleurer la diversité et la complexité de l'œuvre de Foucher et de ses contemporains.

\section{Présentation du fonds Alfred Foucher à la Société asiatique ${ }^{8}$}

\section{Historique}

Grâce aux précieuses indications de M. Pierre-Sylvain Filliozat et Mme Jeanne-Marie Allier, bibliothécaire de la Société asiatique depuis le début des années $1980^{9}$, on peut ainsi reconstituer l'historique du fonds. Alfred Foucher, mort veuf et sans enfant, légua l'essentiel de ses biens matériels à des cousins et neveux de sa famille ou de celle de sa

7 Dans une présentation qu'il donne de lui-même, au moment de la Première Guerre mondiale, Foucher décrit sa mission de 1895-97 comme une " œuvre d'archéologue et d'explorateur".

8 Sur la Société asiatique et ses fonds d'archives - dont un inventaire global a été également opéré en mai 2007 par mes soins -, voir A. FenET, P.-S. FilliozAT, È. Gran-AyMERICH, "La Société asiatique, une société savante au cœur de l'orientalisme français", Les Nouvelles de l'archéologie 110, nov. 2007 [numéro spécial (dir. A. Schnapp et alii) consacré au programme AREA-France et aux fonds d'archives français intéressant l'histoire de l'archéologie], p. 51-56.

9 La fille du sinologue Paul Demiéville, qui aujourd'hui, bien qu'à la retraite, assure encore bénévolement ses fonctions à la bibliothèque, m’a éclairée sur les aléas récents de la société savante. Je tiens à la remercier chaleureusement pour sa patience, sa générosité et les joyeuses évocations de ses souvenirs d'enfance ou de famille. 
femme ; pour ce qui est des documents scientifiques, son testament 10 attribua « à la Société asiatique de Paris et à l'Institut de Civilisation indienne de l'Université de Paris les livres de ma bibliothèque présentant un intérêt philologique ou archéologique ${ }^{11}$ ", et à Jean Filliozat, institué exécuteur testamentaire, «mes manuscrits, notes, documents de caractère philologique, ainsi que ceux de ma femme et ceux de M. É. Senart encore en ma possession (pour être en grande majorité détruits), ainsi que les photographies archéologiques (clichés, diapositifs et tirages sur papier) pour en disposer à son gré ». Heureusement pour nous, tous les papiers du domicile de Foucher ${ }^{12}$ ne furent pas détruits par Jean Filliozat et furent déposés à la Société asiatique à une date indéterminée, dans ses locaux de l'Institut de France. Ils y restèrent jusqu'en 1989, date à laquelle la bibliothèque fut transférée dans les locaux actuels. C'est à cette occasion que les archives furent conditionnées dans les cartons de déménagement dans lesquels nous les avons trouvées en 2006. D’autres documents, apports de la famille Filliozat, conservés dans des petites boîtes archives, sont venus compléter le fonds vers 2003 : ils proviennent semble-t-il de Jean Filliozat qui les avait prélevés dans les papiers Foucher après sa mort ${ }^{13}$.

L'examen et l'inventaire systématiques nous ont laissé deviner des interventions faites à l'intérieur du fonds : des amorces d'identifications ou de classement, des annotations. Il est cependant difficile de repérer précisément les perturbations subies par les documents par rapport à leur état initial à la mort de Foucher, notamment les déplacements ou extractions de papiers à l'intérieur de dossiers ou de liasses, même si

10 M. Pierre-Sylvain Filliozat qui, suite à nos interrogations, a retrouvé le testament dans les papiers de son père (une version autographe et une version dactylographiée légèrement différente, les deux signées), en a fait don, en 2006, à la Société asiatique afin qu'il vienne compléter le fonds Foucher.

11 La bibliothèque de la Société asiatique conserve un cahier intitulé "legs A. Foucher" qui recense apparemment les ouvrages ayant appartenu à Foucher et les doublons éventuels avec des livres de la S. A. Aucune date ni nom n'apparaît dans cette liste; il n'y est fait par ailleurs aucune mention de papiers ou d'archives.

12 Alfred Foucher et son épouse habitaient une maison à Sceaux (15, rue du Maréchal Joffre) où ils s'étaient installés en 1927 après leur mariage et leur longue pérégrination en Asie ; la taille et l'ancienneté du logement expliquent en partie l'importance volumétrique et chronologique du fonds. Une clause du testament concernant " tous mes effets et objets personnels, [...] papiers d'état-civil, parchemins universitaires ou honorifiques, photographies des familles Michel et alliées, photographies de voyage de caractère privé etc. " légués à un petit-neveu laisse supposer qu'un tri a cependant été opéré dans les papiers après la mort de Foucher.

13 Il s'agit notamment du manuscrit du livre posthume de Foucher, Les vies antérieures du Bouddha d'après les textes et les monuments de l'Inde. Choix de contes présentés par A. Foucher, Membre de l'Institut, et illustrés par Janine Auboyer, Conservateur au Musée Guimet, Paris, 1955 (Publications du Musée Guimet, Bibliothèque de diffusion LXI), que J. Filliozat a fait éditer. 
quelques études reproduisant des lettres ou photographies du fonds Foucher nous en donnent quelques indices ${ }^{14}$.

\section{Description physique}

Le fonds Alfred Foucher contient approximativement tous les documents accumulés par le savant au cours de sa vie et gardés auprès de lui. Ils sont actuellement conservés à la bibliothèque de la Société asiatique, sise au 52 rue du Cardinal Lemoine, à Paris Ve (dans un immeuble du Collège de France), dans des cartons divers constituant un volume d'environ $1 \mathrm{~m}^{3}$ de documents papiers compactés ${ }^{15}$, auxquels s'ajoutent 10 cartons de tailles diverses contenant des plaques photographiques.

Notre première phase de travail a permis le dépouillement et l'inventaire systématiques de tous les cartons contenant les documents papier ${ }^{16}$ - nous n'avons pas

14 Pour son article sur la DAFA, M. Paul Bernard (P. BERNARD, "L'œuvre de la Délégation archéologique française en Afghanistan (1922-1982)”, CRAI 2002, p. 1287-1323) a examiné le fonds, à la recherche de documents sur la DAFA et les fouilles de Bactres, en particulier la correspondance entre Émile Senart et Foucher et les plaques photographiques. Par ailleurs, l'ouvrage récent de G.M. Bongard-Levine, R. LARDinOIS, A.A. Vigasin, Correspondances orientalistes entre Paris et Saint-Pétersbourg (1887-1935), Paris, 2002 (Mémoires de l'Académie des Inscriptions et Belles Lettres XXVI), publie quelques lettres et photos du fonds ; M. Roland Lardinois m’a confirmé avoir regardé rapidement le fonds Foucher à la recherche de documents sur Saint-Pétersbourg. Quelques annotations disparates font supposer que $\mathrm{M}^{\mathrm{me}}$ Francine Tissot l'a consulté (en partie ou totalement ?) - au milieu des années 90 semble-t-il - en laissant ici ou là quelques remarques générales (type : énoncé d'un contenu sur une chemise ou sous-chemise) mais pas toujours correctes, ainsi qu'une liste dactylographiée (trouvée dans le dossier " manuscrits " du bureau de la bibliothécaire) datée de mars 1996 identifiant " un paquet de photographies, sans doutes offertes à M. Foucher par J. Barthoux, et concernant principalement le site de Hadda en Afghanistan ".

15 Au début de notre travail, en janvier 2006, nous avons trouvé dix-sept cartons entièrement remplis (format : $53 \mathrm{~cm} \mathrm{lg}$ x $35 \mathrm{~cm}$ large x $25 \mathrm{~cm} \mathrm{ht}$ ) intitulés respectivement : "Foucher 1 " jusque "Foucher 11", "Foucher correspondance", "Foucher photos", "Foucher Lahore", "Foucher? Aymonier ?", ainsi que cinq dossiers carton (format : $33 \mathrm{~cm} \mathrm{lg} \mathrm{x} 25 \mathrm{~cm}$ large x $8 \mathrm{~cm}$ ép.) intitulés pour l'un "Archives Foucher", pour les autres "Legs Foucher". Notre dépouillement a montré que ces appellations diverses - sauf le carton "correspondance", mais il n'est pas non plus le seul à contenir des lettres - ne correspondaient ni à une évolution chronologique des contenus ni à un éventuel contenu thématique des cartons - au mieux, à une des nombreuses chemises contenues dans le carton en question. Au contenu de ces cartons rattachés explicitement à l'indianiste, se sont rajoutés plus tard des petits ensembles d'archives Foucher trouvés mélangés dans des cartons d'autres archives de la Société asiatique.

16 L'inventaire systématique " en l'état" du contenu des cartons a été mené en établissant, sur autant de fichiers Word, une liste détaillée par carton, précisant successivement et si possible : la nature du document (pièce à pièce ou par ensemble de pièces ), ses caracté- 
encore touché aux cartons de plaques photographiques -, et de ce fait la délimitation $\mathrm{du}$ fonds et les principales distinctions thématiques ${ }^{17}$. Parallèlement, nous avons établi une première biographie d'Alfred Foucher, à l'aide des notices déjà publiées mais aussi complétée par les indications données par les archives ; la connaissance précise notamment des dates des déplacements de Foucher sera très utile pour la datation et le classement chronologique d'un certain nombre de documents. Nous avons également dressé une bibliographie exhaustive du savant (y compris les introductions, comptes rendus, notices, éditions, etc.) afin de déterminer dans un avenir proche ce qui est inédit ou non dans le fonds ${ }^{18}$; enfin élaboré un index des personnages dont les noms sont liés aux documents et à la correspondance. Cet index, qui compte plus de 800 noms, de nationalités et de fonctions très diverses, outre les vérifications de lectures et identifications qu'il a permis et permettra encore dans l'examen des pièces, montre la richesse des contacts de Foucher en France comme à l'étranger dans des milieux tant intellectuels ou scientifiques que diplomatiques ou administratifs.

À l'intérieur des cartons, les documents avaient été entassés de façon à occuper tout l'espace disponible. Ils se présentent cependant le plus souvent - mais on trouve aussi des papiers libres " en vrac " - réunis en des sortes de chemises ou des paquets réalisés à l'aide de bouts de ficelle ; ces ensembles portent parfois un intitulé, soit de la main de Foucher ou de celle de sa femme - qui attestent de regroupements opérés par lui - soit plus récent d'une main indéterminée. Cependant, le dépouillement a montré que ces identifications étaient souvent approximatives, ayant sans doute été faites rapidement au vu d'un ou deux documents alors que le paquet n'était pas uniforme. En réalité, les papiers ont été le plus souvent mélangés à l'intérieur des chemises ou des sous-chemises qu'elles contiennent, ce qui laisse présager un ardu travail de reclassement. $\mathrm{Vu}$ le grand nombre de documents, la multiplicité des activités de Foucher, le manque d'ordre effectif à l'intérieur des cartons comme à l'intérieur des sousensembles, la réutilisation fréquente par Foucher de brouillons, l'absence de légendes sur un grand nombre de photos, etc., le travail de réorganisation devra non seulement regrouper les dossiers distribués au hasard dans les cartons par thèmes, mais aussi veiller

ristiques (manuscrit ou dactylographié, langue...), son auteur, son destinataire, sa datation, un résumé des informations, le nombre de pièces ou feuillets, des réflexions critiques sur le document ou l'ensemble de documents (ex. : à rapprocher de tel autre document ou ensemble de documents ; la chemise rassemble des documents sur telle ou telle question). De manière théorique, sur fichier Word, nous avons ainsi esquissé une ébauche de classement du fonds avec répartition des documents dans des rubriques telles que : Jeunesse"; "Cours et conférences" ; "Mme Foucher" ; "Journaux de voyage" ; "Afghanistan/DAFA" ; " EFEO"; "Publications" ; "Travail éditorial" ; etc. Dans la suite du travail, il faudra reprendre et améliorer ce classement thématique, avant de reconditionner le fonds pour le rendre ainsi accessible aux chercheurs et au personnel de la bibliothèque.

18 Cette bibliographie sera intégrée à la publication de la journée Alfred Foucher du 14 décembre 2007 : voir infra, n. 84. 
à définir précisément l'intitulé des dossiers et leur contenu, de façon à ne pas laisser des documents hétéroclites à l'intérieur d'un même dossier.

\section{Les types de documents conservés}

L'essentiel des documents du fonds est à rattacher à la personne d'Alfred Foucher, qui en est soit l'auteur, soit le destinataire. Mais il existe également certaines archives qui ne lui appartiennent pas en propre, mais dont il a pu hériter dans l'optique d'une communication des informations ou d'une transmission des savoirs. Comme l'atteste le testament même de Foucher, ou cette lettre que Louis Finot lui écrivit, le 24 juillet 1927, avant l'un de ses voyages pour l'Indochine : " Je me permets de t'envoyer quelques dossiers que mon prochain départ ne me permet pas de conserver : $1^{\circ}$ Dossier Commission archéologique, qui te revient en qualité de secrétaire [...]. $2^{\circ}$ Dossier des Oeuvres de Barth. Le tome $\mathrm{V}$ et dernier est tout entier en bon à tirer [...]. Je préfere néanmoins ne pas détruire le dossier, parce qu'on peut avoir besoin de s'y reporter pour le règlement final. $3^{\circ}$ Quelques pièces relatives à la Société asiatique et à l'Institut de Civilisation indienne. Les premières te resteront si, comme je l'espère, tu acceptes le secrétariat [...] ", la pratique était courante, dans ce cercle d'orientalistes, de se donner les dossiers ou des éléments de dossier, voire même de la correspondance, comme on le verra plus loin.

Les types de documents conservés dans le fonds sont d'une grande hétérogénéité. On trouve tout d'abord un certain nombre de manuscrits (ouvrages, articles, comptes rendus, conférences, discours, etc.) ${ }^{19}$, sous une forme autographe, parfois dactylographiée, avec pour certains titres également des épreuves corrigées. Pour les ouvrages, il est difficile à l'heure actuelle d'évaluer si les manuscrits ont été conservés dans leur intégralité, car ils se trouvent éparpillés par morceaux : ainsi par exemple, La vieille route de l'Inde est-elle dispersée entre sept cartons dans douze chemises ou ensembles de papiers divers. Par ailleurs le fonds conserve parfois plusieurs versions ou diverses étapes de la réalisation et rédaction d'une même partie d'ouvrage ou d'un article. Il conviendra donc de réunir ce qui doit l'être - et pourquoi pas reconstituer l'ordre des morceaux ou versions. À cet éclatement s'ajoute le problème de la réutilisation de feuilles comme brouillons : ainsi, des passages de manuscrits figurent-ils aux versos de manuscrits antérieurs (ou de textes de conférences, de courriers administratifs, etc.) ${ }^{20}$. Prenons le cas

19 La plume de Foucher fut prolixe : en publications, on ne compte pas moins d'une dizaine de monographies, dont 2 volumes réunissant des cours ou conférences ; la traduction d'un ouvrage allemand ; autour de 80 articles (études, communications, rapports, notices d'encyclopédies...), sans compter les notices nécrologiques, les comptes rendus d'ouvrages, les introductions.

Cette pratique se remarque particulièrement pour la période de la Seconde Guerre mondiale, période durant laquelle Foucher s'est replié avec sa femme loin de Paris, en 
du manuscrit d'un discours de soutien de Foucher à la candidature de Paul Demiéville à l'Académie des inscriptions et belles-lettres ${ }^{21}$ (4 feuillets), placé dans une chemise intitulée "Causeries " regroupant des discours prononcés essentiellement devant ladite assemblée. Sur le verso de la page numérotée " 3 » de ce discours, figure le texte barré d'une autre intervention de Foucher prononcée suite à la conférence d'un orateur indéterminé. Ce feuillet, qui ne peut pas être désolidarisé des trois autres car ensemble ils constituent le manuscrit complet d'un discours donné, offre au verso un fragment, chronologiquement inférieur, d'un discours complètement différent. Ce simple exemple illustre la complexité du catalogage et de l'indexation à établir sur l'ensemble $\mathrm{du}$ fonds si l'on veut que tous les documents soient référencés et facilement consultables.

Les manuscrits et épreuves du fonds n'appartiennent pas tous à Foucher. Pour certains, ils peuvent être rattachés à la personne d'Émile Senart. Le " maître " de Foucher laissa en effet à sa mort (1928) des éditions de textes indiens inachevés, et ce fut Foucher qui, avec l'accord et l'aide des cousins et exécuteurs testamentaires de Senart, M. et $\mathrm{M}^{\mathrm{me}}$ Alphonse Gérard, prit en charge la publication posthume de ces manuscrits. Celle-ci fut réalisée dans une "Collection Émile Senart " créée pour l'occasion, celle des classiques indiens, à l'image de la collection Budé, selon une idée partagée par Foucher et son confrère helléniste de l'Académie Paul Mazon, et à laquelle contribuèrent divers orientalistes sous l'égide de la "Commission Émile Senart 22 ». Si Foucher ne participa au travail d'écriture de tous les volumes de la collection, il garda cependant un regard sur l'ensemble, et c'est pourquoi ses archives contiennent plusieurs manuscrits ou épreuves de textes sanscrits ou palis posthumes de Senart, ainsi que de la correspondance et des notes scientifiques de ce dernier. Le fait est d'autant plus remarquable qu'il n'existe à notre connaissance aucun fonds d'archives Émile Senart ${ }^{23}$. Enfin une troisième catégorie de manuscrits ou épreuves sur des sujets orientalistes provient d'auteurs divers - citons par exemple les premières épreuves du Précis de grammaire

Corrèze, pour fuir les temps troublés et travailler dans son " ermitage »; sur ce repli, cf. le témoignage, daté de 1953, intitulé « Monsieur Alfred Foucher en Corrèze (1940-1942...) ou "un retour à la terre" ", conservé dans le fonds Charles Picard de l'INHA - inventorié et classé par nos soins - sous la référence : INHA, Fonds Ch. Picard 030, carton 7, dossier 5. Le manque de papier, qui s'est prolongé bien après la fin de la guerre, est cause de cette économie.

21 Paul Demiéville a été élu à l'Académie en 1951.

22 Le projet de la "nouvelle collection de Classiques indiens " et les premiers titres prévus sont détaillés dans la plaquette Institut de civilisation indienne 1929-1930, Paris, 1930.

23 Ce que d'ailleurs la correspondance Foucher laisse supposer : celle-ci ne fait part que du don de la bibliothèque Senart à l'Institut de civilisation indienne à la Sorbonne et de cette Commission / Collection Senart. De son côté, M. Roland Lardinois - que je remercie pour cette information - a cherché en vain un hypothétique fonds Senart à l'Institut et auprès de ses descendants. 
palie de Victor Henry (1904) - ; leur présence dans le fonds Foucher s'explique par ses responsabilités éditoriales (notamment dans le cadre de la Commission archéologique de l'Indochine ou de la Délégation archéologique française en Afghanistan), ses amitiés ou soutiens scientifiques.

À tous ces manuscrits et épreuves, se trouvent souvent mêlés des documents divers liés à la réalisation de la publication : iconographie jointe ou indications d'illustrations, lettres et factures échangées avec les éditeurs ou les imprimeurs... Il conviendra de déterminer si ces documents annexes doivent être laissés avec les manuscrits en question, ou au contraire rassemblés avec d'autres archives du même type - c'est le cas notamment de la correspondance avec l'éditeur Van Oest ${ }^{24}$ qui est disséminée dans un très grand nombre de dossiers et de sous-dossiers divers.

Jusqu'ici on a insisté sur les manuscrits d'ouvrages et articles, mais les textes rédigés par Foucher présentent bien d'autres formes : carnets de voyage, tels ceux du voyage en Inde entre 1895 et 1897 25, agrémentés de photos ; nombreux rapports relatifs aux missions de Foucher ou à l'orientalisme, adressés pour un bon nombre au ministère de l'Instruction publique et à celui des Affaires étrangères, au Doyen de l'Université, à l'Académie des inscriptions et belles-lettres ; interventions orales (conférences et dans une moindre mesure cours, participations à des jurys de soutenances de thèses, discours devant des assemblées diverses). Là encore, l'ordre des éléments n'est pas toujours aisé à établir : même si les feuillets d'un même texte ont été numérotés lors de leur écriture par Foucher, ils peuvent se trouver bouleversés et dispersés suite à une réutilisation de sa part (ainsi un texte entier ou un passage extraits d'un ensemble pour la préparation d'une autre conférence, d'un autre cours ou d'une publication) - quand il ne s'agit pas de manipulations postérieures et étrangères.

En dehors des écrits scientifiques de Foucher, une part importante du fonds est constituée par sa correspondance. Celle-ci est doublement intéressante car elle ne consiste pas seulement dans le courrier reçu, mais aussi dans le courrier rédigé par Foucher. En effet, il avait pour habitude de garder les brouillons de ses lettres, avec les corrections éventuelles, souvent seulement allégées des formules finales de politesse. Le texte de ces missives écrites le plus souvent sur des feuilles de cahier (détachées) était ensuite recopié sur du papier à lettre ou éventuellement dactylographié pour être expédié à son destinataire. Pour juger du processus d'écriture de Foucher, il pourrait être intéressant, dans le cas de vraies lettres de sa main conservées dans d'autres fonds, de les comparer avec les lettres-brouillons correspondantes afin de déterminer si le texte de ces dernières était bien définitif. Une fois de plus, la correspondance reçue et envoyée ne se présente pas de façon uniforme : si un gros ensemble se trouvé concentré dans (1935), p. 577-580 et pl. LXXXII. 
quatre dossiers rigides ${ }^{26}$, une bonne partie reste disséminée à travers tous les cartons et ce à l'intérieur de presque toutes les chemises ou sous-ensembles. De surcroît, le plus souvent, les lettres-brouillon - c'est ainsi que nous avons désigné ces documents propres au fonds Foucher - écrites pour des destinataires différents se suivent sur un même feuillet ou un même double feuillet : une seule page recto/verso peut ainsi contenir de une à cinq ou six missives distinctes. Là encore, il conviendra de réfléchir au meilleur système de catalogage et d'indexation pour faciliter les recherches et la consultation des documents. Outre la correspondance d'Alfred Foucher, mentionnons enfin la présence de deux autres ensembles épistolaires bien définis dont il n'est ni l'auteur ni le destinataire : les lettres de Mme Foucher (1919-1926), celles de Jean Commaille à Louis Finot (1908-1913) 27.

Le fonds Alfred Foucher offre aussi un riche éventail d'archives administratives, renvoyant aux multiples activités et responsabilités de la carrière de l'orientaliste ainsi qu'aux nombreuses institutions auxquelles il a appartenu ou avec lesquelles il a eu affaire : depuis l'École française d'Extrême-Orient à la Délégation archéologique française en Afghanistan et à la Maison franco-japonaise ; de la Commission archéologique de l'Indochine à la Commission des fouilles ou au CNRS, de la faculté des lettres de l'Université de Paris à l'Institut de civilisation indienne ; du ministère de l'Instruction publique et ses bureaux au ministère des Affaires étrangères ; l'Académie des inscriptions et belles-lettres et ses commissions, etc. - sans compter quelques feuilles d'imposition, des reçus bancaires, factures ou notes de frais. Certaines pièces peuvent dépasser le cadre strict du calendrier ou des activités que Foucher a dû mener durant ses divers postes à responsabilité : pour illustration nous renvoyons à la lettre de Louis Finot citée supra. Pour en finir avec les archives écrites du couple Foucher, signalons encore la présence de quelques documents de jeunesse : notes de cours, spécimens de vieilles dissertations ou de leurs premiers mémoires d'études.

La documentation photographique quant à elle, d'un volume assez important, se divise en deux catégories : les tirages papier et les plaques photographiques. En ce qui concerne ces dernières, l'examen est encore à faire ; outre le problème du conditionnement et de la conservation, la manipulation de ces objets nécessite des attentions particulières que nous avons préféré insérer dans une phase ultérieure du travail, après l'observation de tous les documents papier ${ }^{28}$. Les photographies proprement dites conservées dans le fonds, pour un certain nombre, sont l'œuvre de Foucher lui-même et pour les autres des vues qu'on lui a fait parvenir (collègues, amis, collectionneurs,

26 Ces quatre dossiers, constitués par Foucher, se trouvaient réunis dans le carton "Correspondance".

27 Pour plus d'information sur ces deux correspondances, voir infra.

28 La Société asiatique conserve une dizaines de cartons de taille diverses de plaques photographiques, certaines avec la mention Foucher. L'examen des plaques, après celui du fonds papier, devrait faciliter la vérification de l'attribution de celles-ci à Foucher, leur identification et leur rapprochement avec des tirages papier éventuels. 
institutions), de manière spontanée ou à la demande de Foucher, soit pour examen de pièces ou faits archéologiques ou pour étude iconographique d'objets, soit pour ses multiples publications. La majorité de ces photos papier ne portent pas de légende, ou alors seulement des commentaires pratiques destinés à l'imprimeur (taille du document à réduire, cadre ou découpage à faire, numéro de figure, etc.). Elles se répartissent de manière anarchique dans le fonds : ou pêle-mêle dans les cartons ; ou formant des paquets pour certains cohérents et d'autres non ; ou dans des chemises à sujet thématique en liaison avec le motif en question ou au contraire mélangées ici et là avec des choses qui n'ont rien à voir ; ou encore gardées avec la lettre dont elles proviennent. On observe également la présence de tirages différents d'un même original qui se trouvent répartis dans différents cartons ou sous-ensembles. Leur état de conservation est variable, du très bon au très mauvais (par ex. quelques unes, portant une légende, sont devenues malheureusement presque invisibles). Du point de vue de la documentation iconographique du fonds, l'ensemble nécessitera un gros travail d'identification et de classement pour distinguer ce qui est réellement inédit de ce qui a déjà été publié ou existe en d'autres exemplaires dans d'autres fonds ${ }^{29}$.

\section{Les archives Alfred Foucher : éclairages thématiques}

Après cette description générale du fonds, il convient à présent de dégager ses principaux centres d'intérêt et d'estimer son importance comme source pour l'histoire de l'archéologie en général et celle de l'orientalisme français en particulier.

\section{La correspondance Alfred Foucher}

La correspondance d'Alfred Foucher conservée ici - rappelons-le : envoyée et reçue représente environ un demi-siècle d'échanges épistolaires avec des destinataires très diversifiés : orientalistes, administrations, politiques et diplomates, membres de la famille et proches..., en France comme à l'étranger. Elle offre non seulement un regard privilégié sur la biographie du savant, sa carrière et son œuvre, mais aussi sur la perception du monde de cet homme cultivé, voyageur, ouvert sur le monde et ses cultures, Français connu et reconnu au niveau international, à la fois témoin et acteur de l'évolution du monde depuis la fin du XIXe s. jusqu'au milieu du XXe s., de la III République avec ses deux guerres mondiales et du début de la IVe, d'une Europe coloniale et d'une Asie en pleine mutation. La correspondance n'offre pas qu'un intérêt scientifique sur les disciplines dans lesquelles Foucher était spécialisé, elle traite de tous les aspects de sa vie et de son époque. Par sa diversité, son étendue géographique, son importance en 
nombre et sa durée, elle représente un ensemble tout à fait exceptionnel pour l'histoire contemporaine et l'histoire des idées.

\section{$\mathrm{M}^{\mathrm{me}}$ Foucher et sa correspondance}

Si nous n'avons fait qu'évoquer jusqu'ici l'épouse de l'orientaliste, cela ne signifie pas qu'il faille la considérer comme un personne insignifiante. Bien au contraire, Eugénie Foucher, née Bazin et appelée par tous ses proches et ami(e)s «Ena», s'est révélée à nos yeux, au fur et à mesure du dépouillement des archives, dotée d'une personnalité peu commune. De 24 ans sa cadette, elle rencontre et cotoie celui qui deviendra son mari lors de ses études d'anglais qu'elle achève vers 1916 par un mémoire sur le poète indien Rabindranath Tagore ; en 1916-1917, on la voit suivre le cours de A. Meillet à l'École pratique des Hautes-Études intitulé "Grammaire comparée de l'Indo-Iranien 30 ». Lorsque Foucher part en mission à Ceylan et en Inde en 1918, elle prend seule le bateau et part le rejoindre pour l'épouser à Colombo le vendredi 14 février 1919. Depuis Ceylan elle le suit avec enthousiasme dans toutes ses pérégrinations en Inde, au Népal, en Perse, en Afghanistan jusqu'au Japon et en Chine, et collabore à tous ses travaux en l'aidant particulièrement dans le domaine de l'intendance, du secrétariat et des langues mortes et vivantes. Même après leur retour en France en novembre 1926, ils restent inséparables jusqu'à sa mort le 30 janvier 1952, neuf mois avant celle d'Alfred ${ }^{31}$.

Par bonheur, le fonds Foucher conserve la correspondance d'Ena durant ces sept années de voyage (1919-1926). L'essentiel des lettres (au total 184 missives !) est adres-

30 Rabindranath Tagore (1861-1941), auteur d'une œuvre prolifique, reçut le prix Nobel de littérature en 1913. Les études d'Ena sur Tagore et ses notes d'indo-iranien sont conservées dans le fonds.

31 Le couple Foucher a co-signé l'ouvrage qui rend compte de l'expérience afghane : FOUCHER, BAZIN-FOUCHER, La vieille route de l'Inde. Ena a publié seule quelques articles spécialisés : "Sur une monnaie du Pancala", in Études d'orientalisme publiées par le musée Guimet à la mémoire de Raymonde Linossier, Paris, 1932, I, p. 145-153 ; "Une réprésentation de Pancika et Hariti à Sanchi”, Journal asiatique 223 (1933), p. 349-350 ; "Le nom scythique de Poséidôn (tel qu'il est rapporté au chap. 59 du L. IV d'Hérodote)", BEFEO 44, 1 (1947-1950 = Mélanges publiés en l'honneur du Cinquantenaire de l'EFEO), p. 13-20 ; "Voyageurs européens en Afghanistan au XIXe siècle" et "L'œuvre de la Délégation archéologique française en Afghanistan", in COLLECTIF, La civilisation iranienne (Perse, Afghanistan, Iran extérieur), Paris, 1952, respectivement chap. IX, p. 307-311 et 316-320 ; un ensemble de comptes rendus : E. Bikerman, Institution des Séleucides, Paris 1938. A. Christensen, L'Iran sous les Sassanides, Copenhague et Paris, 1936. M. Rostovtzeff, Dura-Europos and its art, Oxford, 1938 ; W.W. Tarn, The Greeks in Bactria and India, Cambridge, 1938”, Journal asiatique 230 (1938), p. 501-528; le fonds Foucher conserve également quelques documents (d'elle ou sur elle) qui semblent inédits. 
sée à sa mère, Mme Bazin, et quelques autres à des amies d'enfance, ce à quoi on peut ajouter quelques réponses ( 9 lettres) de la mère à la fille. Cette ensemble épistolaire est doublement intéressant : d'une part parce qu'il met en scène une femme d'archéologue de l'entre-deux-guerres ${ }^{32}$, mais aussi pour la description qu'il donne des missions à la fois scientifiques et diplomatiques successives d'Alfred en Asie durant cette période. Il témoigne tout à la fois de ce que nous pourrions appeler l'" envers du décor " des rapports officiels, des aspects logistiques sur le terrain, de la personnalité et de la place de Mme Foucher dans les travaux de son mari, de la vie des Européens en Asie dans les années 1920, des contacts pris sur place, des recherches scientifiques menées, etc. Dans un autre domaine, il comporte également de précieuses indications pour l'identification des documents iconographiques conservés par ailleurs.

\section{Le bouddhisme et l'Inde, avec ou sans les Anglais}

De formation classique puis sanskritiste, Foucher soutient sa thèse complémentaire sur l'iconographie bouddhique de l'Inde 33 , sujet sur lequel il poursuivra ses recherches durant toute sa vie. Publications diverses, notes scientifiques, correspondance échangée avec d'autres indianistes, photographies : le fonds est composé pour une bonne part de documents relatifs au bouddhisme. Il conserve notamment, du premier voyage du jeune savant en Inde entre 1895 et 1897, ses carnets de voyage agrémentés de photos correspondant au rapport rendu à l'Académie à son retour, ainsi qu'un paquet de lettres : celles envoyées par Foucher à Émile Senart durant cette période. Il y retourne vingt ans plus tard, cette fois à l'invitation du directeur de l'Archaeological Survey of India, Sir John Marshall (1876-1958) 34. D’octobre 1918 à mai 1921, soit durant deux ans et demi, il parcourt l'Inde et le Népal sur les traces de la légende du Bouddha avant de poursuivre en Perse et de là en Afghanistan. Comme il l'affirme dans le texte cité plus haut en introduction, c'est en homme de terrain qu'il connaît non seulement le continent indien mais aussi sa gestion par la couronne britannique, se permettant -

32. On ne peut s'empêcher de faire un rapprochement - bien qu'elles soient de nature très différente - avec les mémoires d'Agatha Christie sur sa vie au Moyen-Orient avec l'archéologue britannique Max Mallowan, dont une traduction française est récemment parue sous le titre La romancière et l'archéologue, Paris, 2005.

33 A. FOUCHER, Étude sur l'iconographie bouddhique de l'Inde d'après des textes inédits. Thèse complémentaire présentée à la faculté des lettres de l'Université de Paris, Paris, 1905.

34 G.N. DAS, "John Marshall 1876-1958", American anthropologist 61, 6 (déc. 1959), p. 1071-1074 ; P. DemiÉVILle, "Éloge funèbre de Sir John Marshall”, CRAI 1959, p. 1417 ; LECLANT (dir.), Second siècle, t. III, p. 469-470 ; sur sa nomination au poste de directeur de l'ASI, voir l'article de N. LAHIRI, "John Marshall's appointment as director-general of the Archaeological Survey of India : a survey of the papers pertaining to his selection", South Asian Studies 13 (1997), p. 127-139. Les travaux majeurs de Sir John Marshall ont porté sur Taxila et sur Sanchi. 
dans un paragraphe qui ne fut finalement pas prononcé - cette critique sur l'Inde anglaise de la fin du XIXe s. : "Tout le premier j'avais mes griefs et déplorais l'abandon où étaient laissés les antiquités et les monuments historiques de l'Inde. Le vice-roi d'alors, Lord Elgin, le petit-fils du spoliateur des marbres du Parthénon d'Athènes, peut-être pour compenser les excès de zèle de son aïeul, avait supprimé par raison d'économie le Service archéologique et il a fallu attendre pour que celui-ci renaisse l'avènement de Lord Curzon ${ }^{35}$. " De fait, la collaboration étroite et amicale entre Foucher et Sir Marshall - le premier contribua d'ailleurs à faire élire le second comme correspondant étranger de l'Académie en 1936 - et de manière générale avec l'Archaeological Survey of India, tout à fait exceptionnelle dans ces temps de rivalité franco-anglaise en matière d'archéologie, a permis de belles découvertes et de magistrales publications, tel le magnifique ouvrage co-signé sur Sanchi ${ }^{36}$. Les échanges entre les deux hommes ont consisté aussi à confronter et mettre en commun leurs observations et leurs expériences en matière de conservation du patrimoine 37 ; c'est ainsi que Foucher s'est trouvé notamment chargé de l'aménagement de la section archéologique du musée de Calcutta. Autre archéologue britannique avec lequel il entretint une relation privilégiée, Sir Aurel Stein (1862-1943) 38, le célèbre explorateur de l'Asie centrale. La correspondance de Foucher 39 montre la forte amitié qui liait les deux hommes et qui prit malheureusement fin avec la convention franco-afghane dont l'Anglais ne supporta pas d'être écarté du fait de la clause d'exclusivité française. Néanmoins, leurs vingt années

35 Lord Curzon fut vice-roi des Indes entre 1898 et 1905. F.D.K. BosCH ("Les rapports entre l'Indochine et l'Indonésie", in L. MALLERET, op. cit., p. 85-89) remarque avec justesse la concomitance entre le renouveau de l'Archaeological Survey des Indes qui, " après de longues années d'attente et d'insignifiance, est ressuscitée par l'initiative du vice-roi Lord Curzon et grâce à la direction énergique de Sir John Marshall ", la création et le développement de l'École française d'Extrême-Orient et ceux du Service archéologique (néerlandais) en Indonésie.

36 Sir J. MARShall, A. FOUCHER, The monuments of Sanchi, with the texts of inscriptions edited, translated and annoted by N. G. Majumdar, Calcutta, 1939, 3 vol.

37 Sir John Marshall a publié sur le sujet un Conservation manual. A handbook for the use of archaeological officers and others entrusted with the care of the ancient monuments, Calcutta, 1923.

38 G. DupOnt-Ferrier, "Éloge funèbre de Sir Aurel Stein", CRAI 1943, p. 612-613 ; È. GrAN-AYMERICH, Dictionnaire biographique d'archéologie 1798-1945, Paris, 2001, p. 648649 ; J. MiRSKY, Sir Aurel Stein, archaeological explorer, Chicago, 1977 ; A. WALKER, Aurel Stein, pioneer of the Silk Road, Londres, 1995 ; J. LECLANT (dir.), Second siècle, t. III, p. 672. Hongrois de naissance, il fut naturalisé britannique en 1904 ; comme Sir John Marshall, il fut élu correspondant de l'Académie, mais six ans avant que Foucher luimême n'y entra.

39 On pourra compléter les lettres des archives de la Société asiatique par celles conservées à l'Académie des sciences de Budapest (soit 54 lettres de Foucher à Stein, et 18 lettres de Stein à Foucher) : E. APOR, H. WANG (éd.), Catalogue of the collections of Sir Aurel Stein in the Library of the Hungarian Academy of Sciences, Budapest, 2002, p. 304. 
de collaboration scientifique ont sans nul doute influencé leur pensée et leur œuvre scientifique respectives.

De manière plus générale, les archives Foucher témoignent ainsi d'un demi-siècle d'indianisme français ou franco-anglais, mais aussi de cinquante ans de relations entre le savant français et les archéologues et érudits indiens. Elles rendent compte également de l'évolution de l'Inde contemporaine en marche vers son indépendance, sans oublier celle du regard des Européens de la première moitié du XXe s. sur le pays et la culture indienne.

\section{L'École française d'Extrême-Orient}

Alfred Foucher dirigea à deux reprises l'École française d'Extrême-Orient : une première fois par intérim, en 1901, durant le congé de Louis Finot rentré en France, la seconde fois en tant que titulaire, entre janvier 1905 et octobre 1907, date à laquelle il fut rappelé à Paris par ses charges d'enseignement. En 1901, l'EFEO, dixit Foucher " la dernière née et la plus exotique des trois filles spirituelles de notre Compagnie [i.e. l'Académie], après les Écoles d'Athènes et de Rome 40 » en est encore à ses débuts, la Mission archéologique de l'Indochine créée fin 1898 venant à peine d'être baptisée EFEO en 1900, avant sa fondation officielle par décret du président Loubet en $1901^{41}$. Elle est alors basée à Saïgon, et l'une des tâches de Foucher fut de préparer le déménagement de la jeune institution à Hanoi qui eut lieu en 1902. C'est Louis Finot qui proposa le nom de son " collègue et ami " pour le remplacer durant son absence, certain du fait que son suppléant " fera profiter l'archéologie indo-chinoise de sa connaissance approfondie de l'art indien et trouvera dans nos monuments d'origine hindoue un objet d'étude digne de sa pénétrante érudition 42 ». Foucher représenta l'École lors du

40 "Discours de M. Alfred Foucher, Membre de l'Institut", in L. MALLERET, Le cinquantenaire de l'EFEO, Paris, 1953, p. 76-79. Ce discours, prononcé à l'Académie des inscriptions et belles-lettres, le 21 mars 1952 et qui constitua la dernière intervention publique de Foucher, émut tous les auditeurs par l'évocation des souvenirs de l'orateur et de ses compagnons disparus de l'EFEO auxquels il rendit hommage, parlant « au nom de ceux que, pour la plupart, je reste seul, ou presque seul, à avoir personnellement connus et que je vais bientôt rejoindre ".

41 Voir à ce sujet les deux "Discours de M. Louis Malleret, directeur de l'EFEO" (à Saïgon, le 3 novembre 1951, puis à Hanoï, trois jours plus tard ), in MALLERET, Cinquantenaire, p. 10-13 et 16-19; sur l'histoire générale de l'École, deux publications récentes à l'occasion de son centenaire : C. ClÉmentin-Ojha, P.-Y. MANGUin, Un siècle pour l'Asie. L'EFEO, 1898-2000, Paris, 2001 ; P. SingARAVÉLOU, L'EFEO ou l'institution des marges (1898-1956). Essai d'histoire sociale et politique de la science coloniale, Paris, 1999.

42 L. FinOT, Rapport à M. le gouverneur général sur les travaux de l'EFEO pendant l'année 1900, Saïgon, 1901, p. 8. 
Congrès des orientalistes de Hambourg en septembre 1902 43. Lors de son mandat officiel en $1905^{44}$, Foucher devient donc le deuxième directeur de l'École et son Art gréco-bouddhique du Gandhâra figure parmi les premiers titres des Publications de l'EFEO. Il contribue à créer la revue de l'École, le Bulletin de l'École française d'ExtrêmeOrient, et collabore activement aux six premiers numéros. Même s'il n'y revint jamais, l'ancien directeur continua toute sa vie à participer de loin à la vie de l'institution et à ses publications tant philologiques qu'archéologiques ${ }^{45}$. Le fonds Foucher contient nombre de documents (archives administratives et scientifiques, correspondances, manuscrits, épreuves, photographies, etc. ) relatifs à l'EFEO et à son évolution durant son premier cinquantenaire. L'intérêt de telles sources n'avait d'ailleurs pas échappé à Louis Finot qui déjà en 1931 écrivait à Foucher, après avoir relu de vieilles lettres concernant l'EFEO et se souciant de leur préservation :

Me mettant à la place du futur historien de l'École française, je me dis qu'il serait bien content de trouver ces pages vivantes pour animer le squelette des documents officiels. Il y a notamment une série de lettres de Maitre qui est vraiment remarquable. Il y en a une autre de M.A. Foucher qui ne l'est pas moins, quoique sur un autre ton. Je compte envoyer à Coedès la correspondance de Maitre 46 pour les archives de l'École, en lui recommandant toutefois de la garder sous clef pendant quelques années, car il s'y trouve des jugements singulièrement incisifs sur des personnages qui ne sont pas encore passés de l'autre côté. Verrais-tu un inconvénient à ce que je lui envoie aussi tes lettres? Pour ma part, je n'en aperçois aucun. La précaution susdite ne serait même pas nécessaire, car elles sont écrites avec une discrétion et un tact si parfaits que personne au monde n'y trouverait l'occasion de s'offusquer. Tu peux m'en croire sur parole. D'autre part elles contiennent des renseignements, qui seront un jour très précieux, sur les premières années de l'École, en 1901, 1904 et années suivantes. Mon avis est donc de les incorporer aux archives de l'École ; mais je ne voudrais pas en disposer sans ton assentiment. Dis-moi ce que tu en penses 47 .

43 A. FOUCHER, "Congrès international des Orientalistes de Hambourg", BEFEO 2 (1902), p. 424-432 ; ID., "Note sur les travaux de l'École française d'Extrême-Orient (18991902)", Verhandlungen des XIII. internationalen Orientalisten-Kongresses (Hamburg September 1902), Leiden, 1904, p. 88-90.

44 A. FOUCHER, "Rapport au Conseil supérieur de l'Indochine sur la situation matérielle et les travaux de l'École française d'Extrême-Orient", BEFEO 6 (1906), p. 489-492.

45 Il reçoit par ailleurs le titre de "membre d'honneur à vie " de l'EFEO, par un arrêté du gouverneur général de l'Indochine daté d'avril 1930.

46 Qui fut membre puis devint directeur de l'EFEO avant la Première Guerre mondiale.

47 Il conviendra évidemment, dans le cadre des recherches à venir, de consulter les archives de l'EFEO, afin de préciser l'existence éventuelle de telles sources complémentaires sur Foucher. 
C'est aussi lors de ses séjours en Indochine que Foucher s'intéressa au site javanais de Barabudur ${ }^{48}$, où il travailla en collaboration avec les Hollandais ${ }^{49}$ et en particulier avec le conservateur du site T. Van Erp ${ }^{50}$. Leur intérêt commun portait sur les basreliefs figurés du stûpa et sur les techniques de conservation patrimoniale ${ }^{51}$ - sur ce second point, cette collaboration préfigurait celle de Foucher avec l'Archaeological Survey of India -, ce dont le fonds apporte plus d'un témoignage.

\section{Angkor et la correspondance Jean Commaille}

Autre site concerné particulièrement par les archives Foucher : celui d'Angkor. En effet, le jeune savant s'est aussi penché sur le cas de cet ensemble architectural et il a même pu s'y rendre à plusieurs reprises au cours de sa vie : la première fois en 1900-1901 et la seconde un quart de siècle plus tard, en décembre 1925, lors de son voyage vers le Japon. S’il n’a rien véritablement publié sur Angkor, il a donné de nombreuses conférences sur le sujet 52 et a participé au développement du site en tant que directeur de l'École française d'Extrême-Orient 53 en 1901, puis en 1905-1907. Il a également supervisé, de loin, au sein de la Commission archéologique de l'Indochine, la publication magistrale de cette dernière sur le Bayon ainsi que l'ouvrage de L. Delaporte sur

48 Il publia deux articles sur ce sujet : "Le Buddha inachevé de Bôrô-Budur", BEFEO 3 (1903), p. 78-80 ; "Notes d'archéologie bouddhique", BEFEO 9 (1909), p. 1-50, ainsi qu'un compte rendu : "C. M. Pleyte, Die Buddha-legende in den Skulpturen des tempels von BôrôBudur, Amsterdam, 1901", Revue de l'histoire des religions 50 (1904), p. 124.

Cf. BEFEO 7 (1907), p. 463. Cette collaboration avec les Hollandais vaudra à Foucher 21 ans plus tard un "diplôme de membre d'honneur " de la Société royale des Arts et Sciences de Batavia, le 24 avril 1928 : BEFEO 28 (1928), p. 332-333 et 644 ss.

N.J. KROM, T. VAN ERP, Beschrijving Barabudur, 1920-1931 (Archaeologisch onderzoek in Nederlandsch-Indië III), 2 vol. de texte +3 vol. de pl. Le premier volume Archaeologische beschrijving est signé N.J. Rom, tandis que T. Van Erp signe le second Bouwkundige beschrijving. Foucher est abondamment cité dans le texte et les notes des deux volumes.

51 T. Van Erp a ainsi consacré le neuvième et dernier chapitre à "De restauratie" dans son volume sur Barabudur (p. 440-468).

52 L'une a été publiée sous le titre "Une récente visite aux ruines d'Angkor et les antiquités bouddhiques du Cambodge" dans l'ouvrage réunissant les conférences faites au Japon : A. FOUCHER, Études sur l'art bouddhique de l'Inde, Tokyo, s. d. (vers 1927) ; une autre résumée sous le titre "Conférence de M. A. Foucher", Bulletin archéologique du Comité des travaux historiques et scientifiques 1912, p. ciii-civ. Par ailleurs, la correspondance Foucher et des mentions ici ou là dans diverses revues orientalistes montrent qu'il est intervenu en public plus de deux fois sur le sujet.

53 Voir notamment : A. FOUCHER, "Rapport à M. le gouverneur général sur les travaux de l'École française d'Extrême-Orient pendant l'année 1901”, BEFEO 2 (1902), p. 433-442. 
les monuments du Cambodge 54 . À ce titre, le fonds conserve de nombreux lettres échangées entre les divers orientalistes concernés, photos, plans, épreuves, etc. relatives au site khmer et datables des années 1900-1920. Il contient également une correspondance particulièrement intéressante, celle de Jean Commaille (1868-1916), le premier conservateur d'Angkor ${ }^{55}$, à Louis Finot - qui fut à plusieurs reprises directeur de l'EFEO -, entre 1908 et 1913. Accompagnées des rapports officiels envoyés à l'EFEO, ces lettres décrivent l'aspect scientifique du travail de J. Commaille, mais aussi les aspects officieux et la vie à Angkor au quotidien. Ensemble, ces documents décrivent avec précision l'aménagement du site, les problèmes de restauration et la lutte permanente contre la végétation.

De manière générale, le fonds Alfred Foucher offre de nombreuses indications sur les études khmères, à travers les échanges qu'entretint Foucher avec les experts en ce domaine jusqu'à la fin de sa vie et les archives administratives émanant des diverses commissions auxquelles il appartenait.

\section{La Délégation archéologique française en Afghanistan}

Spécialiste de l'art du Gandhâra depuis sa thèse 56 et après plusieurs missions en Inde, Foucher ne pouvait qu'espérer explorer l'Afghanistan et ses vestiges archéologiques. Au début des années 1920, alors même qu'il se trouve sur le continent indien, le contexte politique offre l'opportunité d'une ouverture des frontières de ce pays 57 . Le roi Amanullah s'entend avec la France pour une collaboration scientifique et les Foucher sont les premiers Français à passer la frontière indo-afghane, le 13 mars 1922, comme ils l'écrivent triomphalement dans une lettre à Mme Bazin, pour la concrétiser. Foucher

54 Le Bayon d'Angkor Thom. Bas-reliefs publiés par les soins de la Commission archéologique d'après les documents recueillis par la mission Henri Dufour avec la collaboration de Charles Carpeaux, Paris, 1910-1914, 2 vol. ; L. Delaporte, Les monuments du Cambodge, 4 fascicules, Paris, 1914-1924.

55 J. Commaille a contribué à la grande publication du Bayon (op. cit.) à laquelle participèrent avec lui Henri Dufour, Charles Carpeaux, George Coédès, Louis Finot ; il publia également le premier guide du site : J. COMMAILle, Guide aux ruines d'Angkor, Paris, 1912. Sur l'histoire de l'archéologie française à Angkor, voir B. DAGENS, Angkor la forêt de pierre, Paris, 1989 (Découvertes Gallimard 64), avec une photographie et quelques informations sur J. Commaille p. 87-88.

56 Les bas-reliefs gréco-bouddhiques du Gandhâra. Thèse présentée à la faculté des lettres de l'Université de Paris, Paris, 1905.

57 Sur la création de la DAFA et le rôle de Foucher : J. HACKIN, L'auvre de la Délégation archéologique française en Afghanistan (1922-1932). I. L'archéologie bouddhique, Tokyo, Maison franco-japonaise, 1933 ; F. OlIVIER-UTARD, Politique et archéologie : histoire de la Délégation archéologique française en Afghanistan (1922-1982), Paris, 1997, en particulier p. 7-89; BERNARD, Euvre. 
négocie et signe la convention qui permet à la Délégation archéologique française en Afghanistan, dont il est aussi le premier directeur, de mener des fouilles dans le pays. Les travaux de la DAFA commencent par des prospections, puis par une longue campagne de fouilles à Balkh, sur le site présumé de l'ancienne Bactres. Foucher, malade et démoralisé par la stérilité archéologique du chantier, demande un congé ; le ministère des Affaires étrangères agrée sa requête, mais pour l'envoyer remplir une nouvelle mission au Japon. Le couple quitte l'Afghanistan à l'automne 1925 pour ce qu'il pense être une absence temporaire, mais il n'y retournera jamais. Foucher resta cependant directeur de la DAFA jusqu'à la Seconde Guerre mondiale et membre de la Commission des fouilles d'Afghanistan. À ce double titre, il suit de près toutes les activités et publications de la délégation ${ }^{58}$. Le fonds constitue donc une source d'informations désormais incontournable pour l'histoire de l'archéologie franco-afghane. Documents administratifs, correspondances, manuscrits, photos, rapports permettent de suivre son évolution durant les trente premières années de son existence. Ils contribuent tout particulièrement à donner un nouvel éclairage sur la genèse du projet, les démarches préalables, sa naissance avec la convention signée en 1922 et les fouilles de Foucher, grâce aux archives inédites que constituent la correspondance entre ce dernier et Emile Senart ou celle d'Ena à sa mère. C'est pourquoi ce thème a été d'ores et déjà choisi comme première étude et publication des archives Foucher 59 ; l'intérêt d'un tel

58 Les Mémoires de la DAFA, dont La vieille route de l'Inde de Bactres à Taxila des Foucher constitue le premier numéro. Outre ce double volume, Foucher a publié sur l'Afghanistan les articles suivants : "Notes sur les antiquités bouddhiques de Haibâk (Turkestan afghan)", Journal asiatique 205 (1924), p. 139-153 ; "Notes sur l'itinéraire de Hiuan-tsang en Afghanistan", Études asiatiques publiées à l'occasion du 25e anniversaire de l'EFEO, 1925, I, p. 257-284 ; "La délégation archéologique française en Afghanistan (oct. 1922, nov. 1925)", CRAI 1927, p. 117-123 ; "Buste provenant de Hadda (Afghanistan) au musée Guimet", Monuments Piot XXX, 1929, p. 101-110 et pl. IX ; "La Nicée d'Afghanistan", CRAI 1939, p. 435-447 ; "Deux Jâtaka sur ivoire provenant des fouilles de Joseph et Ria Hackin au Bêgrâm de Kâpiçî (Afghanistan), 1939”, India Antiqua, Leyden, 1947, p. 124130 et pl. IX ; des contributions importantes à l'ouvrage Coll., Civilisation iranienne (auquel a également participé sa femme, voir supra note 31).

59 Ce projet a été proposé par M. Paul Bernard et soutenu par M. Pierre-Sylvain Filliozat, qui ont obtenu pour nous l'obtention d'une mission de recherche co-financée par l'Académie des inscriptions et belles-lettres, la DAFA et la Société asiatique pour fin 20072008 ; toute notre gratitude leur est acquise, ainsi qu’à MM. Jean Leclant et Roland Besenval pour leur accord et leur bienveillance. Il consiste en un volume, réalisé par nos soins, consacré à la création de la DAFA et à ses activités en Afghanistan - en particulier la fouille de Bactres -, à partir de la correspondance Foucher et mettant en évidence le contexte scientifique et politique de l'archéologie au Moyen-Orient dans les années 1920. Il prendra en compte les sources complémentaires : les débats parlementaires des années 1920 concernant l'Afghanistan ; au musée Guimet, les photographies et archives relatives à la DAFA, en particulier le journal de Godart, architecte de la DAFA qui travailla avec Foucher ; Archives nationales, ministère de l'Instruction publique, série F17 (17260/1 et 
sujet a pu apparaître dès 1952, sous la plume de Charles Picard dans l'hommage qu'il rendit au premier directeur de la DAFA :

[...] c'est lui qui, en 1922, prépara et décida aussi de l'organisation des recherches archéologiques dans l'Afghanistan, jusqu’alors fermé ; carrefour, à travers l'Antiquité, des rencontres des civilisations méditerranéennes, avec celles de l'Inde et même de l'ExtrêmeAsie. La convention franco-afghane vient d'être renouvelée, et elle permet chaque année des découvertes historiques intéressantes. Al. Foucher devait diriger la première délégation en 1925, dans le pays où J. Hackin et sa femme, pour ne citer que les disparus, s'illustrèrent : à Begram. Dans la correspondance d'Al. Foucher avec Emile Senart, on trouverait d'ailleurs tout un programme de fouilles, préparé avec une étonnante divination, sur les sites à explorer $[\ldots] 60$

\section{La Maison franco-japonaise et l'Institut français de Kyoto}

De l'Afghanistan, Foucher est envoyé par le gouvernement français au Japon, où il est chargé de prendre la direction provisoire de la Maison franco-japonaise. Fondée en 1925, à l'instigation de Paul Claudel alors ambassadeur à Tokyo, elle est confiée par lui aux bons soins de Sylvain Lévi ${ }^{61}$. Ce dernier n'étant pas libre de venir prendre son poste avant septembre 1926, on demande à Foucher, qui offre le double avantage de se trouver déjà en Asie et d'avoir assumé à plusieurs reprises la prise en charge de jeunes institutions (EFEO et DAFA), d'assurer la vacance. Selon les propres termes de S. Lévi, " mon collègue et ami Alfred Foucher, que je suis fier d'appeler mon ancien élève, consentit à assurer l'intérim ; il venait pourtant de passer sept années consécutives, sans relâche, dans l'Inde et en Afghanistan ; il avait créé et organisé le service français des fouilles archéologiques en Afghanistan ; il avait dirigé ou inauguré des fouilles difficiles du fait de la nature et des hommes. Néanmoins, avant de rentrer en France, il accepta

17260/2 ; archives du Service des voyages et missions ; missions individuelles) et AJ 16690 ; archives du ministère des Affaires étrangères à Paris, série Asie-Océanie, Afghanistan 4, 30, 31, 58 ; à l'Académie des IBL, fonds Pelliot (Commission consultative des fouilles en Afghanistan : réf. AREA = FR Arch. IBL / 10 G 7-8), et archives de la Fondation Benoît Garnier concernant la mission 1922-1923 en Afghanistan (réf. AREA = FR Arch. AIBL / 1J 1-7, précisément dossier 1J 6). La publication est prévue pour 2009 dans les Mémoires de l'Académie des inscriptions et belles-lettres, avec une introduction de M. Paul Bernard.

60 PICARD, Alfred Foucher, p. 350.

61 Sur l'histoire de la Maison franco-japonaise, voir Sylvain Lévi et son auvre (Bulletin de la Maison franco-japonaise VIII, 1936, 2-4), Tokyo, 1937, p. 7-62, en particulier l'“avantpropos" de L. JULLIOT DE LA MORANDIÈrE p. 9-11 ; S. LÉVI, "La Maison franco-japonaise de Tokyo", Revue de Paris, 15 septembre 1929, p. 410-428 ; ClÉmentin-OjHA, MANGUIN, EFEO 1898-2000, p. 70-71. 
de faire un long crochet par le Japon, et, comme il le disait d'aller essuyer les plâtres de la maison. Ai-je besoin de dire qu'il y réussit à merveille? Son tact, sa finesse, son élégance d'esprit, sa connaissance profonde de l'art bouddhique étaient mieux à leur place au Japon qu'en Afghanistan 62 [...] ». De fait, tous les talents diplomatiques de Foucher ne sont pas de trop pour finaliser la collaboration franco-japonaise et initier les activités de la Maison. Durant les huit mois passés au pays du Soleil levant (du 21 janvier à la mi-septembre), il déploie toute l'énergie dont il est capable pour la mise en route de l'institution et promouvoir l'image de la France par une série de conférences données à travers tout le Japon. Documents administratifs et correspondances diverses dévoilent les débuts de la Maison, ainsi que celle de sa petite sœur l'Institut français de Kyoto - autrement désigné sous le nom d'Institut franco-japonais du Kansai - créé en 1927. Les lettres échangées avec le géographe Francis Ruellan, qui fut avec P. Claudel 63 l'initiateur du projet et en devint le premier directeur jusqu'en 1930, évoquent les premières années difficiles de l'Institut et les démêlés de Francis Ruellan avec Sylvain Lévi.

\section{Enseignement, communication et transmission des savoirs}

Nommé chargé de cours en 1907 à la faculté des lettres de Paris pour l'enseignement des langues et littératures de l'Inde puis titulaire en 1929 de la chaire des langues et littératures de l'Inde à la Sorbonne, Foucher a assuré - sauf bien sûr pendant les périodes durant lesquelles il se trouvait en mission à l'étranger - jusqu'à sa retraite en 1936 cet enseignement en parallèle avec celui de l'École pratique des Hautes Études, où il avait débuté à partir de 1895, avant d'être nommé en 1914 directeur d'études. À cette double charge s'ajoutent les conférences données à Paris : notamment à l'École normale des jeunes filles à Sèvres, au Musée Guimet, à l'École coloniale ${ }^{64}$, à la radio à la demande du "ministère de l'Information, Radiodiffusion française " et à la Société d'instruction et d'éducation populaires de Sceaux. Pour ce qui est du contenu, il semble avoir ouvert la voie à " l'archéologie bouddhique » dans un enseignement supérieur qui

S. LÉVI, op. cit., p. 419-420.

Cf. L. Garbagnati (éd.), Paul Claudel. Correspondance diplomatique Tokyo 1921-1927, Paris, 1995, p. 48 ss., 61-63, 324-325, 375, 383, 392-399.

64 Sur l'École coloniale, voir B. GRAND, Le 2 avenue de l'Observatoire, de l'École cambodgienne à l'Institut national d'administration publique. Un bâtiment mauresque entre le Luxembourg et l'Observatoire, Paris, 1996, notamment (p. 76 ss.) sur la direction de Georges Hardy (1926-1933) qui sollicita Foucher pour des conférences. Les liens sont par ailleurs étroits entre l'École coloniale et l'archéologie orientale : son premier directeur, Etienne Aymonier (1888-1905), entreprit des missions archéologiques au Cambodge, tandis que le septième, Paul Mus (1946-1950), ancien membre de l'EFEO, appartenait au cercle des orientalistes français bien connus de Foucher (p. 89 ss.). 
était jusque la fin du XIXe s. littéraire et philologique ${ }^{65}$. Co-fondateur en 1927, avec Sylvain Lévi et Emile Senart, de l'Institut de civilisation indienne à la Sorbonne, il assuma, à partir de l'année suivante après la mort de ce dernier, la direction de cet institut qui « se donne [...] mission d'éveiller et d'entretenir les vocations scientifiques, d'assurer dans chaque branche d'un domaine toujours plus divers et plus complexe la continuité, la progression du travail érudit ; plus largement, il vise à maintenir le contact avec tous ceux qu'intéresse l'Inde, à servir de trait d'union spirituel entre les deux grands foyers de culture, la France, héritière des traditions occidentales, l'Inde qui détient les vertus de l'Orient : s'efforçant ainsi, pour sa part, à réaliser le programme d'humanisme syncrétique qu'a défini M.S. Lévi, le maitre des études indiennes, dans son livre L'Inde et le Monde 66 ". Le fonds conserve un certain nombre de textes ou plans des cours et conférences qu'il a dispensés, ainsi que des documents administratifs et correspondances relatifs à la création et aux activités de l'ICI. En tant que professeur et fidèle à l'esprit qui a présidé la création de l'Institut, Foucher dirige les études et les mémoires d'un nombre assez important d'étudiants français mais aussi étrangers, ce qui confere sans nul doute un intérêt très original à ces archives intéressant l'histoire de l'enseignement. L'indianiste a connu et encouragé de nombreux étudiants étrangers, en particulier indiens, japonais ou afghans. Il semble toujours prêt à répondre aux demandes de renseignements et d'appuis (recommandations, demandes de bourses, etc.) de ces jeunes ou moins jeunes - notamment des enseignants - qui souhaitent venir étudier à Paris. Foucher, dans ses rapports et courriers envoyés au ministre et à la faculté durant ses missions, pense en terme de "clientèle " : pour lui, une part de son travail consiste aussi à valoriser l'attrait de la France et à augmenter le nombre d'étudiants de l'Université française. Certains de ces étudiants d'âges divers sont devenus des proches du couple : citons par exemple le suédois Helmer Smith, l'indien Hari Chand ${ }^{67}$, Alice Getty 68 ; de son côté, Ena semble avoir elle aussi porté un regard toujours bienveillant sur ce petit monde, dont il serait pertinent de dresser un tableau plus précis (origine des étudiants, leurs sujets de thèse, ce qu'ils sont devenus de retour dans leur pays). Foucher soutient aussi des étudiants français, pour lesquels il est sans doute plus exigeant ; plusieurs documents posent le problème récurrent du niveau d'exigence que l'on doit attendre d'une thèse soutenue par un étudiant étranger par rapport à celle d'un étudiant français.

65 L. FinOt, A. FOUCHER, "L’enseignement du sanscrit à l'École pratique des Hautes Études", Revue internationale de l'enseignement 35 (janvier-juin 1898), p. 488-492

66 Institut de civilisation indienne 1929-1930, Paris, 1930. L'ouvrage de S. Lévi est paru à Paris en 1926.

67 Qui dans toutes ses lettres à Foucher le qualifie respectueusement de "Guru ".

68 Auteur de : Ganesa. A monograph on the elephant faced-god, with an introduction by Alfred Foucher, Oxford, 1936. 
Accueillant envers les étrangers en France, Foucher est aussi ouvert aux expériences internationales. Lui-même part enseigner un trimestre en Amérique à l'université de Columbia, pendant la Première Guerre mondiale, contribue à la création et aux débuts du collège Amanyeh à Kaboul durant sa mission afghane, sans compter les très nombreuses conférences données durant ses voyages en Asie (en particulier en Inde et au Japon) et en Amérique (à l'Exposition universelle et internationale de San Francisco de 1915, sur Angkor) et au cours de déplacements en Europe (Scandinavie, Suisse, Belgique, Allemagne). L'orientaliste ne fait pas que participer au rayonnement culturel de la France, il profite en outre de ces expériences pour étudier les modèles étrangers et les appliquer dans ce qu'ils ont de meilleur : c'est le cas de l'Institut de civilisation indienne, pour lequel Foucher s'est inspiré d'exemples américains ou d'Europe du Nord ${ }^{69}$. Il sert également, lors de son périple entre l'Inde et l'Afghanistan, de rapporteur à Sylvain Lévi qui lui a demandé d'inspecter pour lui les écoles de l'Alliance israélite en Iran 70 ; ce dont il s'acquitte consciencieusement, comme le montre la correspondance conservée dans le fonds.

De manière générale les archives attestent le fait que durant toute sa vie, Foucher a eu à cœur de transmettre ses connaissances et ses découvertes à des publics de nationalité et de niveaux de culture très diversifiés, et qu'il l'a toujours fait de manière intéressante pour ses auditeurs, dans une langue élégante, avec humour et humilité.

\section{Le cercle orientaliste français}

À travers l'ensemble du fonds, apparaît en filigrane un véritable cercle orientaliste français, centré autour des doyens Emile Senart (1847-1928) ${ }^{71}$, spécialiste de philologie et littérature indiennes, l'indianiste Sylvain Lévi (1863-1935) ${ }^{72}$ et Louis Finot (1864-

69 A. FOUCHER, "L'enseignement de l'indianisme à Columbia University", Revue internationale de l'enseignement 70 (janvier-juin 1916), p. 190-195.

70 Sylvain Lévi rejoignit l'Alliance israélite universelle dans les années 1880 et en fut le président de 1920 à sa mort en 1935. Sur le sionisme de Sylvain Lévi, voir les actes du colloque réunis par L. BANSAT-BOUdON et R. LARDINOIS sous le titre, Sylvain Lévi (1863-1935). Études indiennes, histoire sociale (Bibliothèque de l'École des Hautes Études : Sciences Religieuses 130 ; Turnhout, 2007).

71 L. FinOT, "Émile Senart", BEFEO 28 (1928), p. 335-347 ; A. FOUCHER, "Émile Senart", Journal asiatique 212 (1928), p. 5-18 ; G. GLOTZ, "Éloge funèbre de M. Émile Senart", CRAI 1928, p. 60-65 ; J. BACOT, "La vie agissante d'Émile Senart", L'Asie française, juin 1929, p. 185-190 ; A. GUÉRINOT, "Bibliographie des travaux d'Émile Senart", Journal asiatique 218 (1933), p. 1-75 ; V. GOLOUBEW, "Louis Finot et l'EFEO”, Cahiers de l'EFEO 3 (2e trimestre 1935), p. 1-21 ; LEClANT (dir.), Second siècle, t. II, p. 1308-1309.

72 L. RENOU, "Sylvain Lévi et son œuvre scientifique", Journal asiatique 228 (1936), p. 159 ; varii autores, Institut de civilisation indienne 1933-1935, Paris, 1936, p. 15-57 ; Bongard-Levin, LARdinois, Vigasin, Correspondances orientalistes ; BANSAT-BOUdON, LARDINOIS (éd.), Sylvain Lévi. 
1935) 73 , chartiste de formation et attaché quant à lui aux études indochinoises. Quoique de la génération des deux derniers - avec eux il fréquente par exemple les familles Gaston Maspero (1846-1916) et Edouard Chavannes (1865-1918) -, Foucher se retrouve après leur disparition, au milieu des années 1930, pour ainsi dire le nouveau chef de file des orientalistes français : pour n'en citer que quelques uns, les « indochinois » spécialistes d'art khmer George Coédès (1886-1969) ${ }^{74}$ et Philippe Stern (18951979) ${ }^{75}$, conservateur du musée Guimet, les sinologues Paul Pelliot (1878-1945) ${ }^{76}$, Henri Maspero (1883-1945) 77 et Paul Demiéville (1894-1979) ${ }^{78}$, l'indianiste Jean Filliozat (1906-1982) ${ }^{79}$, les archéologues André Godart (1881-1965) ${ }^{80}$, architecte qui travailla successivement en Irak, Afghanistan et Iran, et Joseph Hackin (1886-1941) ${ }^{81}$,

73 A. COVILlE, "Éloge funèbre de M. Louis Finot", CRAI 1935, p. 210-213; V. GOLOUBEW, "Louis Finot", BEFEO 35 (1936), p. 515-550 ; R. DUSSAUD, Notice sur la vie et les travaux de Louis Finot, Paris, 1938 ; J. LECLANT (dir.), Second siècle, t. I, p. 501-502.

74 A. Sarraut, R. Grousset, "M. George Coedès", Cahiers de la Société des amis de l'EFEO 3 (1959), p. 1-31 ; J. Filliozat, "Notice sur la vie et les travaux de M. George Coedès", BEFEO 57 (1970), p. 1-24 ; J. LECLANT (dir.), Second siècle, t. I, p. 303-304 ; L. LABROUSSE (éd.), Langues' O 1795-1995. Deux siècles d'histoire de l'École des langues orientales (17951995), Paris, 1995, p. 257-258 et 267.

75 J. Auboyer, "Philippe Stern (1895-1979)", Arts asiatiques 36 (1981), p. 66-67.

76 J. Filliozat, "Paul Pelliot", Journal asiatique 1946, p. 13-20 ; LABROUSSE, Langues' O 1795-1995, p. 292-293 ; L. ReNou, Notice sur la vie et les travaux de M. Paul Pelliot, Paris, 1950 ; Société asiatique, Paul Pelliot, Paris, 1946 ; LeClant (dir.), Second siècle, t. II, p. 1124-1125.

77 P. DemiÉVILle, "Henri Maspero (1883-1945)”, Journal asiatique 234 (1943-1945), p. 245-263, suivi d'une "Bibliographie", p. 263-280; A. MERLIN, "Notice sur la vie et les travaux de M. Henri Maspero", CRAI 1951, p. 416-426 ; J. LeClaNT (dir.), Second siècle, t. II, p. 967.

78 G. DE JONG, "Bibliographie 1920-1970", in P. DemiÉville, Choix d'études sinologiques (1921-1970) et ID., Choix d'études bouddhiques, Leyde, 1973 ; J. Heurgon, "Éloge funèbre de M. Paul Demiéville", CRAI 1979, p. 140-142 ; M. SOYMIÉ, "Paul Demiéville (1894-1979)", Journal asiatique 1980, p. 1-10 ; J. GERNET, Notice sur la vie et les travaux de Paul Demiéville, Paris, 1986 ; J. Leclant (dir.), Second siècle, t. I, p. 393-394; LABROUSSE, Langues' O 1795-1995, p. 292-293.

79 R. MARICHAL, "Éloge funèbre de M. Jean Filliozat", CRAI 1982, p. 667-670 ; C. CAILlAT, "Jean Filliozat (1906-1982)", Journal asiatique 271 (1983), p. 1-4, suivie d'une "Bibliographie des travaux de Jean Filliozat" par P. Filliozat, p. 5-24 ; P.-S. Filliozat, "Jean Filliozat 1906-1982", BEFEO 73 (1984), p. 1-30 ; J. LECLANT (dir.), Second siècle, t. I, p. $500-501$.

80 R. LeBĖGUe, "Éloge funèbre d'A. Godard", CRAI 1965, p. 453-454 ; A. PARROT, "André Godard (1881-1965)", Syria 43 (1966), p. 157-158 ; GRAN-AYMERICH, Dictionnaire, p. 303-306 ; J. LECLANT (dir.), Second siècle, t. III, p. 295.

81 Joseph et Ria Hackin, couple d'origine luxembourgeoise au service des arts asiatiques et de la France, Luxembourg, 1988 ; OLIVIER-UTARD, Histoire de la DAFA, passim ; J. LECLANT (dir.), Second siècle, t. III, p. 323. 
le fouilleur de Begram. Tout ce petit monde se connait, se cotoie, se croise entre Paris, l'Asie et les institutions françaises à l'étranger (EFEO, DAFA, Maison franco-japonaise...). L'Académie constitue également pour eux un point de ralliement, et chaque nouvelle élection est l'objet de discussions et stratégies afin d'établir un nouvel orientaliste face au groupe des classiques. Les correspondances et archives administratives de Foucher, auxquelles se mêlent des archives scientifiques des intéressés eux-mêmes, esquissent le portrait et l'évolution de ce cercle sur deux générations, de la fin du XIXe s. jusqu'au milieu du XXe siècle 82 .

\section{Histoire des collections}

Enfin, les documents du fonds peuvent apporter une contribution - sans doute modeste mais réelle - à l'histoire des collections, en l'occurrence des musées du Louvre et Guimet, de la Bibliothèque nationale de France (Département des manuscrits orientaux), du Cabinet des médailles, de la bibliothèque Doucet (aujourd'hui INHA). En effet, de ses voyages en Asie, Foucher a ramené des manuscrits, photos, monnaies, objets, déposés auprès de ces établissements et pour certains desquels les archives devraient aider à préciser l'origine ou l'historique. Ainsi, par exemple, une lettre de Philippe Stern à Foucher, datée du 20 février 1919, à propos de photographies de l'Inde, fait le point sur les collections de clichés attestées à cette date, à Paris, dans les diverses institutions et bibliothèques, en particulier celle d'Art et d'archéologie, et en propose un projet nouveau ${ }^{83}$. Par ailleurs, Foucher a été parfois sollicité par des collectionneurs privés, désireux d'en connaître un peu plus sur telle ou telle pièce, demandes dont la correspondance du fonds garde quelques traces ici ou là.

\section{Perspectives}

Les archives Alfred Foucher conservées à la Société asiatique constituent donc, par leur volume et leur diversité, une source désormais non négligeable pour l'histoire de

82 Les archives complémentaires, dans ce domaine, sont à chercher tout particulièrement à l'Institut de France et à la Société asiatique.

83 Dans ce domaine des collections orientales des bibliothèques parisiennes, on pourra en outre consulter les lettres de Foucher à René Jean, le premier bibliothécaire de J. Doucet dans les années 1900-1910, conservées à la bibliothèque de l'INHA (fonds René Jean, autographes 143, dossier 2, pièces 225-251) : les 27 lettres, écrites entre 1911 et 1915, évoquent principalement les financements de J. Doucet pour des achats de livres et des prises photographiques à Angkor et ailleurs en Asie ; cf. également le Bulletin de la Commission archéologique de l'Indochine 1913, 1, p. viii-x et xiii, séances du 6 juin et du 5 décembre 1912, qui traite de photographies d'Angkor à la Bibliothèque d'art et archéologie Doucet, avec retranscription d'une lettre de René Jean. 
l'orientalisme au sens large. Loin de se cantonner aux seuls domaines des langues et civilisations orientales, elles touchent également l'histoire de l'archéologie et des collections, l'histoire de l'enseignement, l'histoire des sociétés savantes (telles la Société asiatique, l'Académie des inscriptions et belles-lettres pour la France, mais aussi leurs homologues étrangères), l'histoire diplomatique, l'histoire coloniale de la première moitié du XXe s. pour les empires anglais et français, les écoles et instituts français à l'étranger (DAFA, EFEO, Maison franco-japonaise, Instituts franco-japonais de Kyoto), la vie quotidienne et intellectuelle durant les deux guerres mondiales, une grande partie de l'Asie (Ceylan, Inde, Iran, Afghanistan, Indochine - i. e. Cambodge, Laos, Vietnam -, Java, Japon), et les sites tels Angkor, Barabudur ou Bactres (pour ne citer que les principaux). Devant un tel ensemble, on ne peut qu'admirer la complexité de la carrière de Foucher, souligner la polyvalence du savant qui lui permit de passer d'une institution et/ou d'une fonction à une autre, dans le contexte politique, scientifique et diplomatique de la IIIe République.

Le travail initié sur le fonds Foucher doit à court et moyen terme se poursuivre par trois actions concrètes : d'une part une journée d'études consacrée à la mémoire de l'orientaliste sur le thème "Bouddhismes d'Asie. Monuments et littératures ", organisée par M. P.-S. Filliozat, qui se tiendra le 14 décembre 2007 à l'Institut de France ${ }^{84}$; dans le cadre de la fin du programme AREA, les reconditionnement, classement et catalogage général sous forme de notices des archives; enfin la réalisation d'un ouvrage sur la création et les débuts de la DAFA (voir supra).

À plus long terme, on espère que le fonds contribuera à une étude plus globale de l'histoire de l'archéologie, celle de la pratique de l'archéologie française à l'étranger sous la IIIe République et de son évolution après la Seconde Guerre mondiale, en considérant tout à la fois ses méthodes, ses dimensions internationales (projets politiques ou coloniaux, rapports avec l'archéologie étrangère - particulièrement anglaise ou allemande - considérée sous l'angle de l'émulation, de l'imitation, de la collaboration ou de la rivalité), son évolution sur à peine plus d'un siècle, son héritage et ses permanences dans l'archéologie d'aujourd'hui, ses liens avec l'enseignement et la muséographie. L'examen des archives Foucher conduit en particulier à une mise en perspective des écoles archéologiques et instituts français à l'étranger : la Maison franco-japonaise à Tokyo - " une institution scientifique de recherches dans le genre de nos écoles d'Athènes ou de Rome, mais conçue selon une formule nouvelle de collaboration à frais communs ", écrit Foucher dans une lettre de 1929 à l'indianiste Paul Oltramare -, l'Institut franco-japonais de Kyoto, l'École française d'Extrême-Orient -présentée par Foucher comme la "sœur cadette " de l'EFA, dans une lettre de 1933 à George

84 Au programme, une dizaine de communications sur l'œuvre de Foucher et sa place dans l'histoire du bouddhisme. Nous y donnerons pour notre part une présentation générale de la correspondance de Foucher, complétée par une bibliographie exhaustive des travaux du savant et de son épouse. 
Coedès -, la Délégation archéologique française en Afghanistan, et par comparaison la Délégation scientifique en Perse, les Écoles françaises d'Athènes et de Rome et l'Institut français d'archéologie d'Istanbul ${ }^{85}$. Au-delà de l'histoire particulière des institutions, il mène à une réflexion globale sur leur genèse, leurs modèles et leurs fonctionnements administratifs, sur la spécificité de leurs activités scientifiques, sur leurs acteurs et sur les rapports entretenus par l'administration et les savants français avec leurs homologues étrangers et les autorités locales. De la Méditerranée à l'Extrême-Orient, c'est comme si l'histoire de ces institutions montrait la quête et son dépassement, par la France de la III ${ }^{e}$ République, de la route d'Alexandre. Du moins, en ce qui concerne le parcours personnel d'Alfred Foucher, tant intellectuel qu'archéologique, c'est bien l'itinéraire qui a été suivi.

\begin{tabular}{l} 
Annick FENET \\
\hline Programme AREA \\
11, rue d'Alsace \\
F-78100 St-Germain-en-Laye \\
annick.fenet@mae.u-paris10.fr
\end{tabular}

85 Sur l'histoire de ces institutions et de l'archéologie, nous renvoyons aux trois ouvrages de référence : È. GrAN-AYMERICH, Naissance archéologie (pour Athènes, Rome et la Perse) ; Chevalier, Recherche Moyen-Orient (Istanbul, Perse, Afghanistan) ; Olivier-Utard, Histoire de la DAFA (Afghanistan, comparaison avec la Perse p. 37 ss.). 


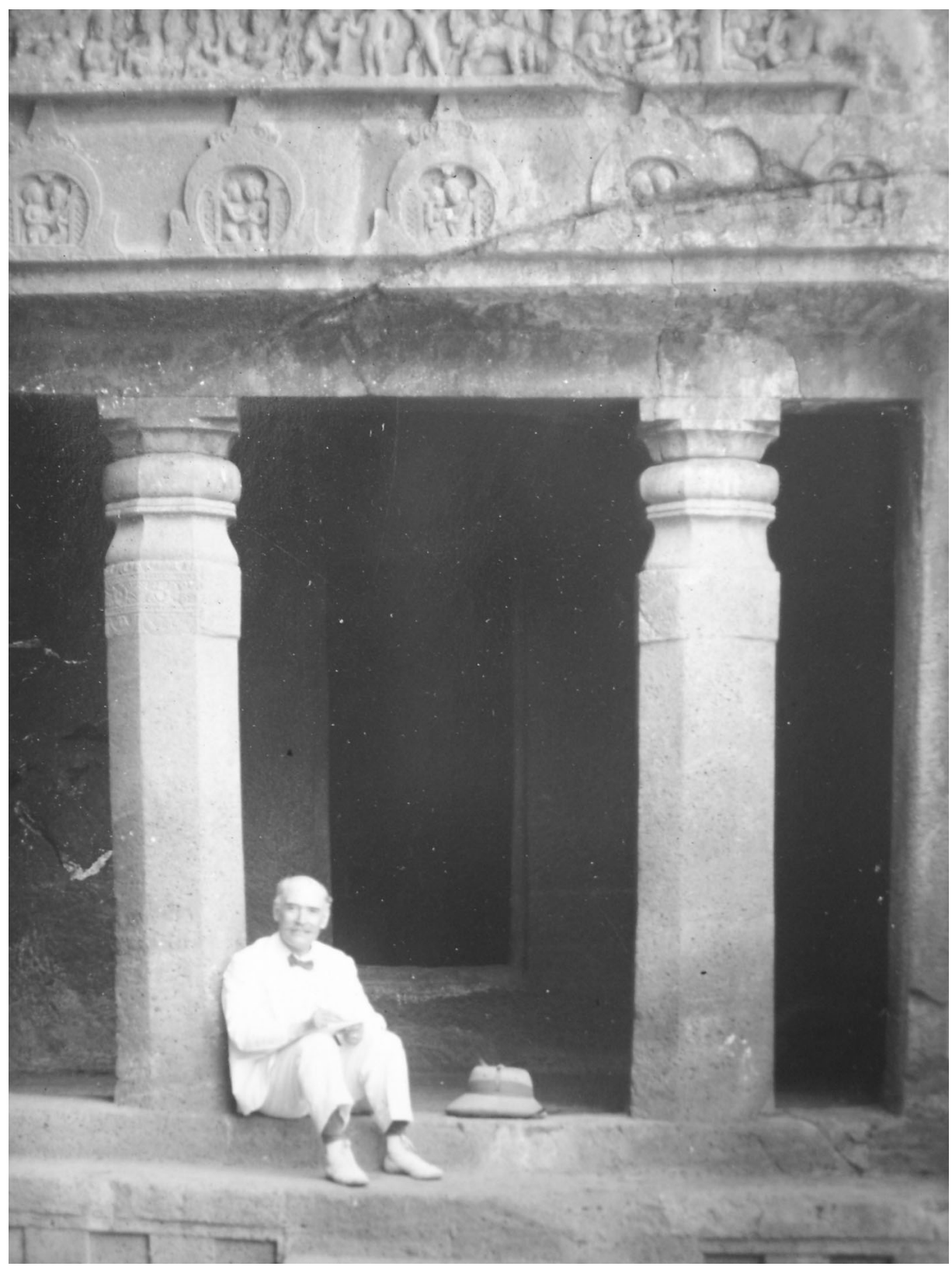

Alfred Foucher à Ajanta (Inde), janv.-fév. 1920.

Archives A. Foucher (C) Société asiatique 


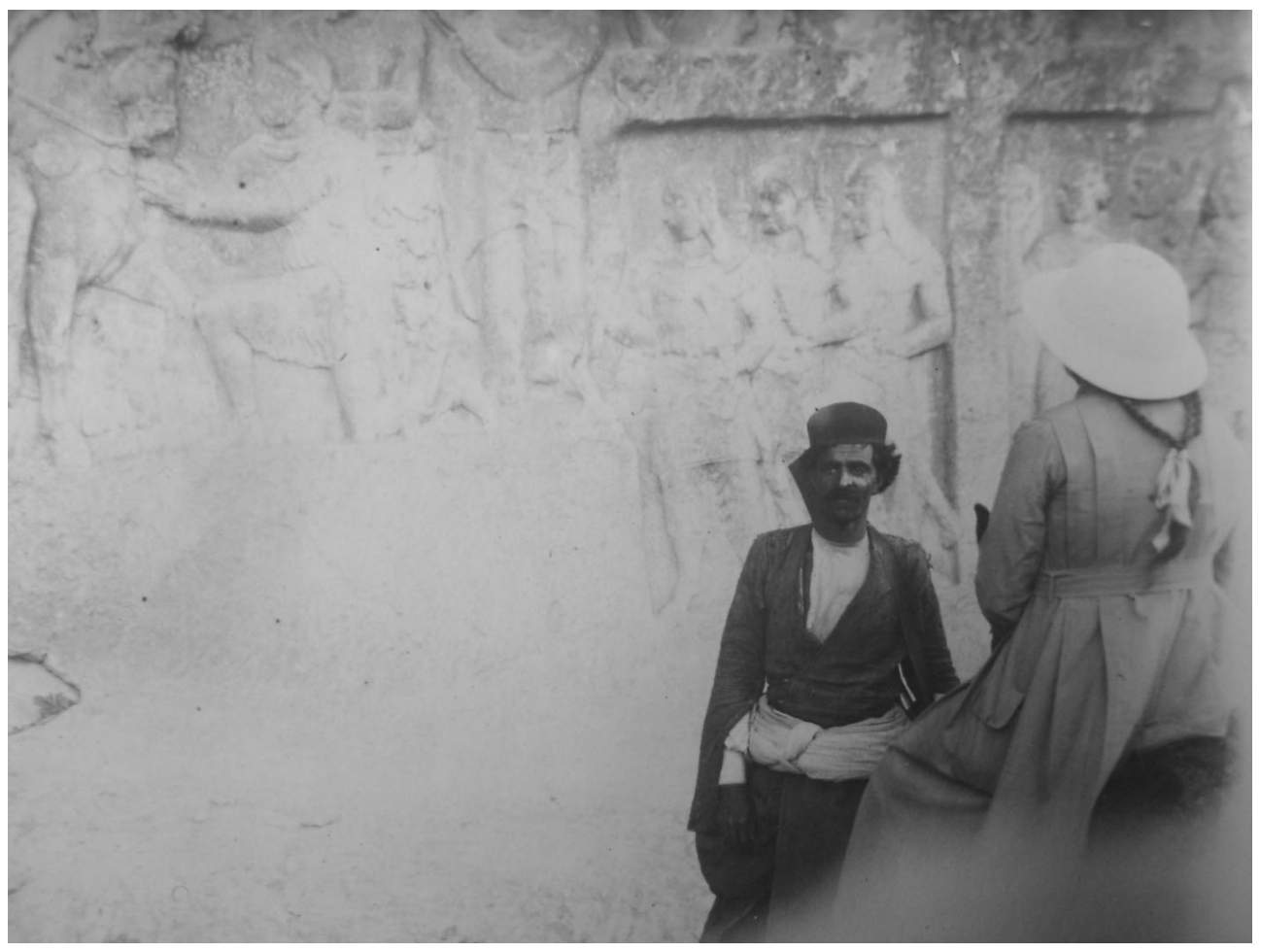

Mme Foucher en Perse (été 1921).

L'un des bas-reliefs rupestres : soumission de l'empereur Valérien à Shapour.

Ena sur sa mule le contemple. Un « tufangchi » la garde.

Archives A. Foucher (C) Société asiatique 